Original Research Paper

\title{
Machine Maintenance Design using Markov Chain Method to Reduce Maintenance Costs
}

\author{
Akhmad Syakhroni', Rizka Fajar Adi Darmawan', Novi Marlyana1 \\ ${ }^{I}$ Industrial Engineering, Faculty of Industrial Technology, Universitas Islam Sultan Agung. \\ Semarang, Indonesia.
}

Article History

Received:

15.04.2021

Revised:

25.05.2021

\section{Accepted:}

09.06.2021

*Corresponding Author:

Akhmad Syakhroni

Email:

syakhroni@unissula.ac.id

This is an open access article, licensed under: $\mathrm{CC}-\mathrm{BY}-\mathrm{SA}$
Abstract: PT. XYZ is a company that focuses on construction with ready mix concrete product (cast). The problem faced by the company is that the schedule is not suitable for machine maintenance activities so that it still results in high maintenance costs incurred by the company. By using the markov chain method can plan maintenance time in order to reduce downtime so as to minimize maintenance costs. The results obtained by the proposal for the company are for proposal I it takes 49.78 hours $=50$ hours at a cost of Rp. $16,984,605$, the cost savings of Rp. $73,545,395$ (81.24\%). Schedule for each machine such as wheel loaders every 14,009 hours, batching plant machines every 16.604 hours, truck mixer machines every 19,168 hours. Scheduling the second proposal will take 26.62 hours $=27$ hours at a cost of Rp. 9,080,664, the cost savings of Rp. 81,449,336 (89.97\%). Schedule for every machine such as wheel loaders every 7.490 hours, batching plant machines every 8.877 hours, mixer truck machines every 10.248 hours. Judging from the results obtained, the recommendation given is $P 3$ (corrective maintenance at status 4 and preventive maintenance at status 2,3$)$.

Keywords: Maintenance, Markov Chain, Scheduling. 


\section{Introduction}

PT. XYZ is a company that focuses on the construction sector with products produced in the form of Ready Mix Concrete (cast) for concrete. Based on land work in collaboration with state-owned and private-owned projects. Its activities include leveling, forming and stockpiling housing complex land, construction of toll roads, dams and irrigation canals, construction of reclamation mining roads and river normalization, construction of canals, airports, ports, industrial estates, power plants and others. The use of sophisticated machines is one of the solutions used by this company because using sophisticated machines can simplify the production process. The machines used for the production process are Truck Mixer, Batching Plant, and Wheel Loader.

In terms of usage, it is very different from one another, to support the smooth production process, the machine must be ready and in prime condition, therefore it is necessary to maintain routine. The maintenance process greatly affects the costs incurred. Machines that are often used if they have poor maintenance will often suffer damage and can interfere with the production process. Maintenance of the machine must use a good method in order to avoid very high maintenance costs.

Table 1. Damage for 1 Year

\begin{tabular}{cccc}
\hline Machine Name & Month & Downtime & Cost \\
\hline Wheel Loader & $\begin{array}{c}\text { December 2018 to } \\
\text { November 2019 }\end{array}$ & 84.92 jam & Rp. 25,08,000 \\
\hline Batching Plant & $\begin{array}{c}\text { December 2018 to } \\
\text { November 2019 }\end{array}$ & 99.75 jam & Rp. 23,006,000 \\
\hline Truck Mixer & $\begin{array}{c}\text { December 2018 to } \\
\text { November 2019 }\end{array}$ & 117.67 jam & Rp. 42,443,000 \\
\hline & Total & Rp. 90,530,000 \\
\hline
\end{tabular}

Judging from the table above, mixer trucks often experience maintenance, but the distance between the wheel loaders, batching plant and truck mixer engines is not that much, therefore to overcome these three machines a maintenance policy will be planned because these machines have a high level of damage. Of these three types of machines, the engine failure always changes every month. If maintenance is not done, the machine will be damaged, but if maintenance is done too often it will result in cost overruns that must be incurred by the company. If higher maintenance costs will cause losses to the company, the company wants to minimize maintenance costs. At least maintenance costs can fall between $20 \%-35 \%$ of costs before scheduling.

The category of engine failure is divided into 3, namely minor damage, moderate damage, severe damage. In lightly damaged, the production equipment continues to work properly even though suddenly there are problems with production. For moderate damage, the machine can usually operate but is in an alarming state. Meanwhile, for heavy damage, the production equipment cannot be used so that production has stopped. Maintenance of production machines is useful for minimizing the damage that will occur suddenly and later can guarantee that the machine continues to work without experiencing a decrease in performance. Therefore, the importance of a machine maintenance design strategy that is carried out on a scheduled basis will reduce machine failures during production. In order to propose improvements to the machine maintenance policy, it is necessary to use precise calculations where the calculations can predict changes that are likely to occur in the future. Calculation with the right method is expected to provide a solution in designing a structured maintenance machine that can minimize maintenance budgets.

\section{Literature Review}

To minimize maintenance costs, a company must re-schedule maintenance. This maintenance is in the form of intervals of periodic checking activities that are rescheduled or checking components that are considered fatal and very disturbing if there is damage. For this reason, it is necessary to reschedule using the markov chain method in order to minimize the maintenance budget such as research with the research title "maintenance planning of the saos milling pump machine with the markov chain method to minimize maintenance costs (case study: PT. Lombok Gandaria, maintenance unit))" with the 
results of the research is that the mill pump will be maintained every 1 month according to the results of observations. This condition is because the machine works every day and only dies when it is not reproducing. Corrective maintenance was carried out in a rather heavy status and the cost was changed to Rp. 11,490, thus saving Rp. 23,897 [1]

Judging from other observations with the title "Application of the markov chain method in machine maintenance planning to minimize maintenance costs (case study: PTPN IV adolina business unit, with the result of the research being that maintenance must be carried out periodically so that the production process can run smoothly and without interruption. issued Rp. 912,037,697, after processing the data using the markov chain method, the second proposal was Rp. 124,806,232, so that Rp. 787,231,465 saved and proper maintenance every 0.8 months [2]

From the next observation entitled "The use of the markov chain method in scheduling machine maintenance to minimize the cost of machine damage and maintenance of the 303 mill machine at PT. Steel Pipe Industry Of Indonesia Unit 3 "the results obtained from this study are the budget spent for moderate and severe damaged conditions Rp. 548,437,272. The maintenance budget for the proposed minor damage was Rp. 175,499,992, the difference from the company budget was Rp. 372,937,335 (68\%). Moderately damaged condition is Rp. 241,312,393 with a difference from the company budget of Rp. 307,124,864 (56\%), in severe damage it is Rp. 76,781,215 with a difference from the company budget of Rp. 471,656,042 (86\%). From the results obtained, the savings are very much, so the proposal can be used as a reference in making decisions and the proposed schedule, which is every 0.4 months as a maintenance schedule on condition that there must be spare spare parts [3]

The next research is research with the research title "machine maintenance planning using the markov chain method at PT. Karyamitra Budisentosa Pandaan". The results obtained from this research are that the proposed maintenance of the stitching machine is in P3 with the action taken is corrective maintenance in severely damaged conditions (status 4) and preventive maintenance on minor and moderate damage (status 2 and 3 ) the result is budget savings. Stitching machine maintenance Rp. 678,600 or $4.9 \%$ of the maintenance cost carried out by the company, from Rp. $13,759,000$ / 6 months to Rp. 13,080,600 / 6 months [4]

The next research is research with the research title "filling machine maintenance planning with the markov chain method to minimize maintenance costs at PT Swabina Gatra." The results obtained from this study are the budget for proposal I Rp. 7,490,472 and need 29 hours of maintenance time, there is a difference of Rp. 14,600,678 (66\%) of the real budget of IDR 22,091,150. The budget for proposal II was IDR 528,364 and it took 2 hours to make a difference of IDR 21,562,787 (97\%) from the real budget of IDR 22,091,150. As a result, the proposal given to the company is proposal II [5]

From the study literature it has been explained that the appropriate method is the markov chain method. Because by using this method the researcher can schedule machine maintenance according to the calculation of each proposal and at the same time can calculate the minimization of costs incurred if the company implements a machine maintenance scheduling system that is calculated using the Markov chain method, where the results of direct cost minimization can be seen because already taken into account if the scheduling is implemented in the company.

\subsection{Basis of Theory}

\subsubsection{Machine Maintenance}

Maintenance is an activity carried out to maintain tools or machines that support the production process with the aim that the machine can operate optimally without any disturbance. Maintenance activities allow production support facilities to run optimally without decreasing performance while the machine is running. So that the production process is not constrained and in accordance with the results that have been previously planned.

Apart from not being constrained by the production process, routine maintenance activities can also generate good profits for the company, namely reducing the cost of replacing damaged components [1]. Activities undertaken include:

1. activity checking (checking).

2. cleaning activity (cleaning).

3. lubrication activity.

4. activity to fix or repair (repairing).

5. activities to replace spare parts (replacement).

6. Calibration activity of machine tools. 


\subsubsection{Types of Maintenance}

The Types of Maintenance are:

1. Preventive maintenance

Preventive maintenance or often known as calendar based maintenance is maintenance with the intention of preventing damage or in other words preventive care before serious damage occurs [2].

2. Corrective maintenance

Machine maintenance that aims to increase the efficiency of the machine or the engine repair process because it is damaged that causes component replacement. This activity is carried out only if there is damage. So that the machine can be used again to support the production process.

3. Maintenance Running

Maintenance is carried out when the production machine is in the production process.

4. Predictive Maintenance

Machine maintenance functions to find out if the machine is malfunctioning or is damaged.

5. Breakdown Maintenance

Maintenance performed with the function of repairing or repairing that is planned after the appearance of a fault. To support maintenance must prepare spare part components and experts.

\subsubsection{Classification of Damage Conditions}

In data processing, it will later calculate the transition probability, where before the calculation is carried out the machine conditions are grouped into 4 groups. The classification is as follows:

1. Good condition

Equipment is said to be in good condition if the equipment can perform its function as it can be used to carry out the production process without a failure. To keep the machine in good condition, the company must carry out preventive maintenance regularly and regularly. This good condition is also called status 1 .

2. The condition of minor damage

Equipment is said to be in a condition of minor damage if the equipment can still perform its function or can still operate, but at any time there is damage or interference in carrying out its function. The disruption or damage that occurs is relatively small so that the maintenance budget is not too much. This condition of minor damage is also known as status 2 .

3. Condition of moderate damage

The equipment is said to be in a moderate condition if the equipment can still function or can still operate, but the condition of the machine is worrying. This condition of minor damage is also known as status 3 .

4. Severe damage conditions

Equipment is said to be in a condition of serious damage if the equipment cannot perform its function or cannot operate. If serious damage occurs, the production process stops and the machine repair process can take a long time. Repair activities also cost a lot. Severe damage condition is also known as status 4 [6].

\subsection{Markov Chain}

The markov chain or known as the markov process is a stochastic system which has the property that the occurrence of a state in a period depends on and only on the state. Data obtained periodically according to certain time intervals according to place is also information about events that change with time. By using the markov chain, we can predict the steps (movements) from one event to the next with probability. The Markov chain is a mathematical technique whose function is to carry out design modeling of various systems and business processes. This technique is useful for predicting changes in the future in the form of dynamic variables from the past. Markov chains are often used in maintenance problems. The stochastic process stage $\{\mathrm{Xt}\}(\mathrm{t}=0.1, \ldots)$ is a markov chain if it has markovian properties. The conditional probability $\mathrm{P}\{\mathrm{Xt}+1=\mathrm{j} \mid \mathrm{Xt}=\mathrm{i}\}$ for the Markov chain is called the transition probability (one step) or commonly called the n-step transition probability. To make writing easier, it is written in matrix form [7], [8], [9].

The stochastic process stage $\{\mathrm{Xt}\}(\mathrm{t}=0.1, \ldots)$ is a markov chain if it has markovian properties. The conditional probability $\mathrm{P}\{\mathrm{Xt}+1=\mathrm{j} \mid \mathrm{Xt}=\mathrm{i}\}$ for the Markov chain is called the transition probability 
(one step) or commonly called the n-step transition probability. To make writing easier, it is written in matrix form.

Table 2. Probability of N-Step Transitions

\begin{tabular}{|c|c|c|c|c|}
\hline State & 0 & 1 & $\ldots$ & $\mathrm{M}$ \\
\hline 0 & $\mathrm{Pij}_{1 j}^{(n)}$ & $\mathrm{Pij}^{(+12)}$ & $\ldots .$. & $\mathrm{Pij}^{[n]}$ \\
\hline 1 & $\mathrm{P}_{i j}(n)$ & $\mathrm{Pij}^{(n)}$ & $\ldots . .$. & $\mathrm{P}_{1 j}[n]$ \\
\hline$\cdot$ & . & . & $\ldots .$. & \\
\hline . & . & 8 & $\ldots .$. & \\
\hline $\mathrm{M}$ & $\mathrm{Pij}(n)$ & $\mathrm{Pij}[n]$ & $\ldots$. & $\mathrm{Pij}^{[n]}$ \\
\hline
\end{tabular}

\begin{tabular}{|c|c|c|c|c|}
\hline \multicolumn{2}{|c|}{ State } & 0 & 1 & $\ldots \mathrm{M}$ \\
\hline \multirow{3}{*}{$\mathrm{P}^{(n)}=$} & 0 & $\mathrm{P}^{(n)}$ & $\mathrm{P} 01^{(n)}$ & $\ldots \mathrm{P} 0 \mathrm{~m}^{(n)}$ \\
\hline & 1 & $\mathrm{P} 10^{(n)}$ & $\mathrm{P} 11^{(n)}$ & $\ldots \mathrm{P} 1 \mathrm{~m}^{(n)}$ \\
\hline & M & $\stackrel{\cdots}{\mathrm{PmO}^{(n)}}$ & $\operatorname{Pm}_{1}^{(n)}$ & $\ldots \mathrm{Pmm}^{(n)}$ \\
\hline
\end{tabular}

For $\mathrm{n}=0,1,2, \ldots$ or, the equivalent of an $\mathrm{n}$-step transition matrix

Note that the transition probability for a given row and column is for the transition from the row state to the column state. When $\mathrm{n}=1$, we discard the superscript $\mathrm{n}$ and call it just a transition matrix, or it can be represented as a matrix,

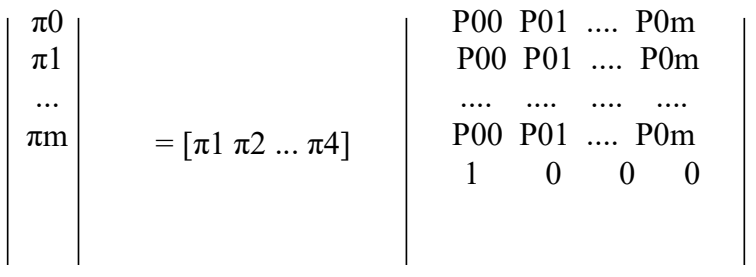

$$
\begin{aligned}
& \pi 0=\pi 0 \mathrm{P} 00+\pi 1 \mathrm{P} 01+\ldots .+\pi \mathrm{MPM} 1 \\
& \pi 1=\pi 0 \mathrm{P} 01+\pi 1 \mathrm{P} 11+\ldots . .+\pi \mathrm{MPM} 2 \\
& \ldots . \quad=\ldots \ldots . .+\ldots \ldots . .+\ldots . .+\ldots \ldots . . \\
& \pi \mathrm{M}=\pi 0 \mathrm{P} 0 \mathrm{M}+\pi 1 \mathrm{P} 1 \mathrm{M}+\ldots . .+\pi \mathrm{MPMM} \\
& 1 \quad=\pi 0 \quad+\pi 1 \quad+\ldots \ldots+\pi \mathrm{M}
\end{aligned}
$$

Decision making is useful for providing what actions to take if a sudden breakdown occurs in the production equipment [10]. In making decisions must be in accordance with the circumstances that are happening. The state or condition of the machine must be in accordance with what decisions to make [7]. After the inspection, the machines or production equipment are grouped as show in Table 3. 
Table 3. Criteria for Machine Condition

\begin{tabular}{ll}
\hline \multicolumn{1}{c}{ Status } & \multicolumn{1}{c}{ Condition } \\
\hline 1 & Good \\
\hline 2 & Lightly Damaged \\
\hline 3 & Moderately Damaged \\
\hline 4 & Heavily Damaged \\
\hline
\end{tabular}

The decisions taken in determining treatment are as show in Table 4.

Table 4. Types of Decisions

\begin{tabular}{cl}
\hline Decision & Action \\
\hline 1 & Take no action \\
\hline 2 & $\begin{array}{l}\text { Perform preventive maintenance (system returns to its previous } \\
\text { state) }\end{array}$ \\
\hline 3 & Perform corrective maintenance (system returns to status 1) \\
\hline
\end{tabular}

Table 5. Care Policy

\begin{tabular}{clcccc}
\hline Wisdom & \multicolumn{1}{c}{ Note } & $\mathrm{d} 1(\mathrm{P})$ & $\mathrm{d} 2(\mathrm{P})$ & $\mathrm{d} 3(\mathrm{P})$ & $\mathrm{d} 4(\mathrm{P})$ \\
\hline P1 & $\begin{array}{l}\text { Corrective maintenance at status 4 and } \\
\text { preventive maintenance at status 3 }\end{array}$ & 1 & 1 & 2 & 3 \\
\hline P2 & $\begin{array}{l}\text { Corrective maintenance at status 3, 4 and } \\
\text { preventive maintenance at status 2 }\end{array}$ & 1 & 2 & 3 & 3 \\
\hline P3 & $\begin{array}{l}\text { Corrective maintenance at status 4 and } \\
\text { preventive maintenance at status 2 and 3 }\end{array}$ & 1 & 2 & 2 & 3 \\
\hline P4 & Corrective maintenance at status 3 and 4 & 1 & 1 & 3 & 3 \\
\hline
\end{tabular}

P1, P2, P3 and P4 are the proposed policies carried out in accordance with the circumstances or conditions.

If a unit is in a lightly damaged and moderately damaged condition, then the unit will not change to good condition or it can be said that a lightly damaged and moderately damaged unit will remain in a lightly damaged or moderately damaged condition or will move into a heavily damaged condition. If the unit is in a badly damaged condition or the unit deteriorates until the next inspection stage, or the unit has not been repaired and will be repaired at the next stage of the inspection. From that statement it can be described with a closed set schematic as show in Figure 1.

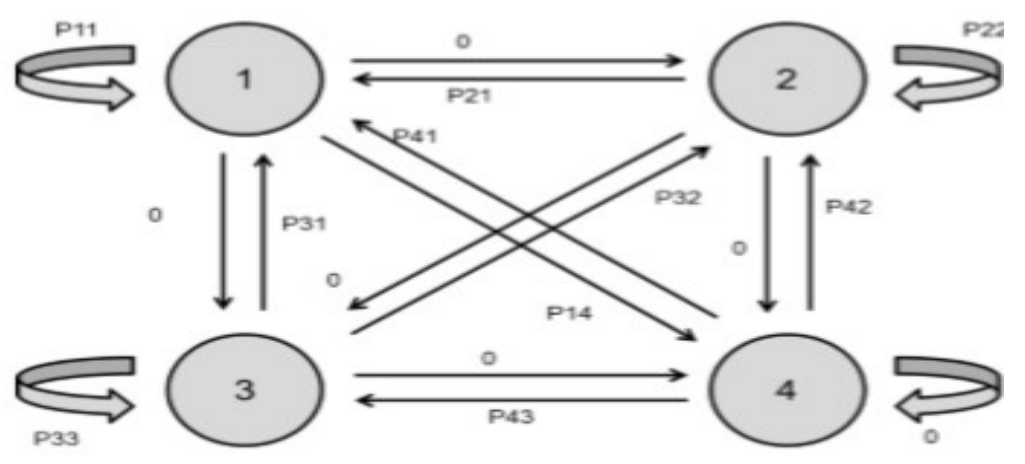

Figure 1. Schematic of a Closed Set (Close Set) 
Note:

1. stating condition or status 1 (good)

2. stating condition or status 2 (slightly damaged)

3. stating the condition or status 3 (moderate damage)

4. declare condition or status 4 (heavily damaged)

In accordance with the previous opinion it can be explained that the machine has a transition probability Pij, which states that if a machine is in status i then it will move to status $j$ next time. From that statement it is usually written in tabular form as show in Table 6.

Table 6. Probability of Damage

\begin{tabular}{ccccc}
\hline \multicolumn{1}{c}{ Final status (j) } & & & & \\
Initial status (i) & $1(\mathrm{j})$ & $2(\mathrm{j})$ & $3(\mathrm{j})$ & $4(\mathrm{j})$ \\
\hline 1 & & & & \\
\hline 2 & $\mathrm{P} 11$ & $\mathrm{P} 12$ & $\mathrm{P} 13$ & $\mathrm{P} 14$ \\
\hline 3 & 0 & $\mathrm{P} 22$ & $\mathrm{P} 23$ & $\mathrm{P} 24$ \\
\hline 4 & 0 & 0 & $\mathrm{P} 33$ & $\mathrm{P} 34$ \\
\hline
\end{tabular}

Note: $\quad 1 \rightarrow$ if it is repaired

$0 \rightarrow$ if not repaired

After determining the status probability, a transition will be determined which can be calculated from the total proportion of components undergoing a status transition. Then formed into an initial transition matrix or proposed maintenance $(\mathrm{P} 0)$

Table 7. Item Transition Probability for December 2018 - November 2019

\begin{tabular}{|c|c|c|c|c|c|c|c|c|c|c|}
\hline \multirow{2}{*}{ Month } & \multicolumn{10}{|c|}{ Status } \\
\hline & P11 & P12 & P13 & P14 & P22 & P23 & P24 & P33 & P34 & P44 \\
\hline \multicolumn{11}{|l|}{ Dec18 } \\
\hline \multicolumn{11}{|l|}{ Jan19 } \\
\hline \multicolumn{11}{|l|}{ Feb19 } \\
\hline \multicolumn{11}{|l|}{ Mar19 } \\
\hline \multicolumn{11}{|l|}{ Apr19 } \\
\hline \multicolumn{11}{|l|}{ May19 } \\
\hline \multicolumn{11}{|l|}{ Jun19 } \\
\hline \multicolumn{11}{|l|}{ Jul19 } \\
\hline \multicolumn{11}{|l|}{ Agst19 } \\
\hline \multicolumn{11}{|l|}{ Sept19 } \\
\hline \multicolumn{11}{|l|}{ Oct19 } \\
\hline \multicolumn{11}{|l|}{ Nov19 } \\
\hline Total & & & & & & & & & & \\
\hline
\end{tabular}

After determining the status probability, a transition will be determined which can be calculated from the proportion of the total components undergoing a status transition.

The item-i one-step matrix is a proposed maintenance show in Table 8. 
Table 8. Item-0 One-Step Transition Matrix

\begin{tabular}{|c|c|c|c|c|}
\hline \multicolumn{5}{|c|}{$\mathbf{j}$} \\
\hline $\mathrm{i}$ & 1 & 2 & 3 & 4 \\
\hline 1 & P11 & P12 & P13 & P14 \\
\hline 2 & 0 & P22 & P23 & P24 \\
\hline 3 & 0 & 0 & P33 & P34 \\
\hline 4 & 1 & 0 & 0 & 0 \\
\hline
\end{tabular}

$\pi \mathrm{j}>0 \quad \pi \mathrm{j}=\Sigma_{j-1}^{4} \llbracket \pi \mathrm{j} P \mathrm{ij}$ for $\mathrm{j}=1,2,3,4 \rrbracket$

$\Sigma_{j-1}^{4} \llbracket \pi j=1 \rrbracket$

$\Sigma_{j}^{4} \llbracket \pi j=1 \rrbracket$

$$
\left|\begin{array}{l}
\pi 1 \\
\pi 2 \\
\pi 3 \\
\pi 4
\end{array}\right|=\left[\begin{array}{llllll}
\pi 1 & \pi 2 & \pi 3 & \pi 4
\end{array}\right] \quad\left|\begin{array}{cccc}
\mathrm{P} 11 & \mathrm{P} 12 & \mathrm{P} 13 & \mathrm{P} 14 \\
0 & \mathrm{P} 22 & \mathrm{P} 23 & \mathrm{P} 24 \\
0 & 0 & \mathrm{P} 33 & \mathrm{P} 34 \\
\mathrm{P} 41 & 0 & 0 & 0
\end{array}\right|
$$

Note : $\pi 1+\pi 2+\pi 3+\pi 4=1$

Then the followig equation will be obtained:

\begin{tabular}{|c|c|c|}
\hline $\begin{array}{ll}\pi 1 & + \\
\mathrm{P} 11 & \pi 1+\end{array}$ & $\pi 2+$ & $\begin{array}{l}\pi 4 \\
\mathrm{P} 41 \pi 4\end{array}$ \\
\hline $12 \pi 1+$ & $\mathrm{P} 22 \pi 2$ & \\
\hline $13 \pi 1+$ & $\mathrm{P} 23 \pi 2+\mathrm{P} 33 \pi$ & \\
\hline $14 \pi 1+$ & $\mathrm{P} 24 \pi 2+\mathrm{P} 34 \pi$ & \\
\hline
\end{tabular}

\subsection{Markov Chain Maintenance Planning}

In order to obtain more efficient maintenance and reduce maintenance budgets. Then four engine maintenance plans will be proposed which will be chosen if they have the smallest budget, from the four proposals, among others, are as follows [11] [12] [13]:

1. Corrective maintenance at status 4 and preventive maintenance at status 3 .

2. Corrective maintenance at status 3 and 4 and preventive maintenance at status 2 .

3. Corrective maintenance at status 4 and preventive maintenance at status 2 and 3 .

4. Corrective maintenance at status 3 and status 4 .

\section{Methodology}

Determination In determining the maintenance budget, the cost of corrective maintenance and preventive maintenance is taken at the time of maintenance and emphasizes downtime costs. With the preventive maintenance scheduling, it can reduce corrective maintenance and emergency maintenance budgets so that the issued budget does not swell [14] [15].

The following are the budgets that occur if there is a maintenance plan or not in a company [16], [17]:

1. Downtime costs 
Because there is a system that does not perform its function optimally due to improper maintenance schedules resulting in a loss of company profits, this is called downtime costs. Which is usually downtime, among others, operator costs, loss of results from production and others.

2. Corrective Maintenance Budget

Damage is a condition in which a device or system is unable to perform its task as its function, so that no output is issued. The damage resulted in an additional budget that had to be spent for repairs. To overcome this, there must be a regular maintenance schedule so that no damage occurs at any time. The corrective maintenance budget is written in $\mathrm{C} 2 \mathrm{i}$ notation for every ith item.

$\mathrm{C} 2 \mathrm{i}=($ average downtime per month $\mathrm{X}$ downtime per hour $)$

3. Preventive Maintenance Budget

The budget will be spent on preventive maintenance. The budget depends on the number of machines that will be inspected and repaired. Downtime costs are costs that will be sacrificed because with preventive maintenance, production hours will be interrupted by maintenance. The preventive maintenance budget is usually written in the notation $\mathrm{Cli}$ for the item- $\mathrm{i}$ preventive budget.

$\mathrm{C} 1 \mathrm{i}=($ average downtime per month $\mathrm{X}$ hourly downtime costs $)$

4. Budget Average Expectations

In accordance with the preventive maintenance and corrective maintenance budget, a budget will be obtained for each machine. If the results obtained are then multiplied by the state of the feeding, the expected average budget for each treatment is obtained.

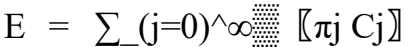

$$
\begin{aligned}
& =\pi 1(\ldots)+\pi 2(\ldots)+\ldots .(\ldots)+\pi \mathrm{M}(\ldots)
\end{aligned}
$$

\section{Finding and Discussion}

\subsection{Machine Data Underwent Status Changes}

\section{a. Wheel Loader Machine}

Table 9. Data on changes to the status of the Wheel Loader

\begin{tabular}{ccccccccccc}
\hline \multirow{2}{*}{ Month } & \multicolumn{10}{c}{ Condition (Status) } \\
\cline { 2 - 12 } & $\mathbf{B} / \mathbf{B}$ & $\mathbf{B} / \mathbf{r}$ & $\mathbf{B} / \mathbf{s}$ & $\mathbf{B} / \mathbf{b}$ & $\mathbf{r} / \mathbf{r}$ & $\mathbf{r} / \mathbf{s}$ & $\mathbf{r} / \mathbf{b}$ & $\mathbf{s} / \mathbf{s}$ & $\mathbf{s} / \mathbf{b}$ & $\mathbf{b} / \mathbf{B}$ \\
\hline December & 0 & 0 & 0 & 0 & 0 & 0 & 1 & 0 & 0 & 0 \\
\hline January & 0 & 0 & 0 & 0 & 1 & 0 & 0 & 0 & 0 & 0 \\
\hline February & 0 & 0 & 0 & 0 & 0 & 0 & 0 & 0 & 0 & 1 \\
\hline March & 0 & 0 & 0 & 0 & 0 & 0 & 0 & 0 & 1 & 0 \\
\hline April & 0 & 0 & 0 & 0 & 0 & 1 & 0 & 0 & 0 & 0 \\
\hline May & 0 & 0 & 0 & 0 & 1 & 0 & 0 & 0 & 0 & 0 \\
\hline June & 1 & 0 & 0 & 0 & 0 & 0 & 0 & 0 & 0 & 0 \\
\hline July & 0 & 1 & 0 & 0 & 0 & 0 & 0 & 0 & 0 & 0 \\
\hline August & 0 & 0 & 0 & 1 & 0 & 0 & 0 & 0 & 0 & 0 \\
\hline September & 0 & 0 & 1 & 0 & 0 & 0 & 0 & 0 & 0 & 0 \\
\hline October & 0 & 0 & 0 & 0 & 0 & 0 & 0 & 0 & 0 & 1 \\
\hline November & 0 & 0 & 0 & 0 & 0 & 0 & 0 & 1 & 0 & 0 \\
\hline
\end{tabular}




\section{b. Batching Plant Machine}

Table 10. Data on changes to the status of the Batching Plant

\begin{tabular}{ccccccccccc}
\hline \multirow{2}{*}{ Month } & \multicolumn{10}{c}{ Condition (Status) } \\
\cline { 2 - 13 } & $\mathbf{B} / \mathbf{B}$ & $\mathbf{B} / \mathbf{r}$ & $\mathbf{B} / \mathbf{s}$ & $\mathbf{B} / \mathbf{b}$ & $\mathbf{r} / \mathbf{r}$ & $\mathbf{r} / \mathbf{s}$ & $\mathbf{r} / \mathbf{b}$ & $\mathbf{s} / \mathbf{s}$ & $\mathbf{s} / \mathbf{b}$ & $\mathbf{b} / \mathbf{B}$ \\
\hline December & 0 & 1 & 0 & 0 & 0 & 0 & 0 & 0 & 0 & 0 \\
\hline January & 0 & 0 & 0 & 0 & 0 & 0 & 0 & 0 & 1 & 0 \\
\hline February & 0 & 0 & 0 & 1 & 0 & 0 & 0 & 0 & 0 & 0 \\
\hline March & 0 & 0 & 1 & 0 & 0 & 0 & 0 & 0 & 0 & 0 \\
\hline April & 0 & 0 & 0 & 0 & 0 & 0 & 0 & 1 & 0 & 0 \\
\hline May & 0 & 1 & 0 & 0 & 0 & 0 & 0 & 0 & 0 & 0 \\
\hline June & 0 & 0 & 0 & 0 & 0 & 1 & 0 & 0 & 0 & 0 \\
\hline July & 0 & 0 & 0 & 0 & 0 & 0 & 1 & 0 & 0 & 0 \\
\hline August & 0 & 0 & 0 & 0 & 0 & 0 & 0 & 0 & 0 & 1 \\
\hline September & 0 & 0 & 0 & 0 & 0 & 0 & 0 & 1 & 0 & 0 \\
\hline October & 1 & 0 & 0 & 0 & 0 & 0 & 0 & 0 & 0 & 0 \\
\hline November & 0 & 0 & 0 & 0 & 0 & 0 & 1 & 0 & 0 & 0 \\
\hline
\end{tabular}

\section{c. Truck Mixer Machine}

Table 11. Data on Changes to the Status of the Truck Mixer

\begin{tabular}{ccccccccccc}
\hline \multirow{2}{*}{ Month } & \multicolumn{10}{c}{ Condition (status) } \\
\cline { 2 - 12 } & $\mathbf{B} / \mathbf{B}$ & $\mathbf{B} / \mathbf{r}$ & $\mathbf{B} / \mathbf{s}$ & $\mathbf{B} / \mathbf{b}$ & $\mathbf{r} / \mathbf{r}$ & $\mathbf{r} / \mathbf{s}$ & $\mathbf{r} / \mathbf{b}$ & $\mathbf{s} / \mathbf{s}$ & $\mathbf{s} / \mathbf{b}$ & $\mathbf{b} / \mathbf{B}$ \\
\hline December & 0 & 0 & 0 & 0 & 0 & 0 & 0 & 9 & 6 & 0 \\
\hline January & 0 & 0 & 0 & 0 & 7 & 4 & 4 & 0 & 0 & 0 \\
\hline February & 0 & 0 & 0 & 0 & 3 & 8 & 4 & 0 & 0 & 0 \\
\hline March & 0 & 0 & 0 & 0 & 0 & 0 & 0 & 11 & 4 & 0 \\
\hline April & 8 & 0 & 0 & 0 & 0 & 0 & 0 & 0 & 0 & 7 \\
\hline May & 0 & 0 & 0 & 0 & 3 & 7 & 5 & 0 & 0 & 0 \\
\hline June & 6 & 0 & 0 & 0 & 0 & 0 & 0 & 0 & 0 & 9 \\
\hline July & 0 & 0 & 0 & 0 & 8 & 2 & 5 & 0 & 0 & 0 \\
\hline August & 0 & 7 & 5 & 3 & 0 & 0 & 0 & 0 & 0 & 0 \\
\hline September & 0 & 0 & 0 & 0 & 0 & 0 & 0 & 10 & 5 & 0 \\
\hline October & 10 & 0 & 0 & 0 & 0 & 0 & 0 & 0 & 0 & 5 \\
\hline November & 7 & 0 & 0 & 0 & 0 & 0 & 0 & 0 & 0 & 8 \\
\hline
\end{tabular}




\subsection{Calculation of Machine State Transition Probability}

Table 12. Wheel Loader Status Change Data

\begin{tabular}{ccccccccccc}
\hline \multirow{2}{*}{ Month } & \multicolumn{10}{c}{ Condition (status) } \\
\cline { 2 - 14 } & $\mathbf{B} / \mathbf{B}$ & $\mathbf{B} / \mathbf{r}$ & $\mathbf{B} / \mathbf{s}$ & $\mathbf{B} / \mathbf{b}$ & $\mathbf{r} / \mathbf{r}$ & $\mathbf{r} / \mathbf{s}$ & $\mathbf{r} / \mathbf{b}$ & $\mathbf{s} / \mathbf{s}$ & $\mathbf{s} / \mathbf{b}$ & $\mathbf{b} / \mathbf{B}$ \\
\hline December & 0 & 0 & 0 & 0 & 0 & 0 & $1 / 4$ & 0 & 0 & 0 \\
\hline January & 0 & 0 & 0 & 0 & $1 / 4$ & 0 & 0 & 0 & 0 & 0 \\
\hline February & 0 & 0 & 0 & 0 & 0 & 0 & 0 & 0 & 0 & $1 / 2$ \\
\hline March & 0 & 0 & 0 & 0 & 0 & 0 & 0 & 0 & $1 / 2$ & 0 \\
\hline April & 0 & 0 & 0 & 0 & 0 & $1 / 4$ & 0 & 0 & 0 & 0 \\
\hline May & 0 & 0 & 0 & 0 & $1 / 4$ & 0 & 0 & 0 & 0 & 0 \\
\hline June & $1 / 4$ & 0 & 0 & 0 & 0 & 0 & 0 & 0 & 0 & 0 \\
\hline July & 0 & $1 / 4$ & 0 & 0 & 0 & 0 & 0 & 0 & 0 & 0 \\
\hline August & 0 & 0 & 0 & $1 / 4$ & 0 & 0 & 0 & 0 & 0 & 0 \\
\hline September & 0 & 0 & $1 / 4$ & 0 & 0 & 0 & 0 & 0 & 0 & 0 \\
\hline October & 0 & 0 & 0 & 0 & 0 & 0 & 0 & 0 & 0 & $1 / 2$ \\
\hline November & 0 & 0 & 0 & 0 & 0 & 0 & 0 & $1 / 2$ & 0 & 0 \\
\hline Sum & 0.250 & 0.250 & 0.250 & 0.250 & 0.500 & 0.250 & 0.250 & 0.500 & 0.500 & 1 \\
\hline
\end{tabular}

Table 13. Batching Plant Status Change Data

\begin{tabular}{ccccccccccc}
\hline \multirow{2}{*}{ Month } & \multicolumn{10}{c}{ Condition (status) } \\
\cline { 2 - 14 } & $\mathbf{B} / \mathbf{B}$ & $\mathbf{B} / \mathbf{r}$ & $\mathbf{B} / \mathbf{s}$ & $\mathbf{B} / \mathbf{b}$ & $\mathbf{r} / \mathbf{r}$ & $\mathbf{r} / \mathbf{s}$ & $\mathbf{r} / \mathbf{b}$ & $\mathbf{s} / \mathbf{s}$ & $\mathbf{s} / \mathbf{b}$ & $\mathbf{b} / \mathbf{B}$ \\
\hline December & 0 & $1 / 5$ & 0 & 0 & 0 & 0 & 0 & 0 & 0 & 0 \\
\hline January & 0 & 0 & 0 & 0 & 0 & 0 & 0 & 0 & $1 / 3$ & 0 \\
\hline February & 0 & 0 & 0 & $1 / 5$ & 0 & 0 & 0 & 0 & 0 & 0 \\
\hline March & 0 & 0 & $1 / 5$ & 0 & 0 & 0 & 0 & 0 & 0 & 0 \\
\hline April & 0 & 0 & 0 & 0 & 0 & 0 & 0 & $1 / 3$ & 0 & 0 \\
\hline May & 0 & $1 / 5$ & 0 & 0 & 0 & 0 & 0 & 0 & 0 & 0 \\
\hline June & 0 & 0 & 0 & 0 & 0 & $1 / 3$ & 0 & 0 & 0 & 0 \\
\hline July & 0 & 0 & 0 & 0 & 0 & 0 & $1 / 3$ & 0 & 0 & 0 \\
\hline August & 0 & 0 & 0 & 0 & 0 & 0 & 0 & 0 & 0 & 1 \\
\hline September & 0 & 0 & 0 & 0 & 0 & 0 & 0 & $1 / 3$ & 0 & 0 \\
\hline October & $1 / 5$ & 0 & 0 & 0 & 0 & 0 & 0 & 0 & 0 & 0 \\
\hline November & 0 & 0 & 0 & 0 & 0 & 0 & $1 / 3$ & 0 & 0 & 0 \\
\hline Sum & 0.2 & 0.4 & 0.2 & 0.2 & 0 & 0.333 & 0.667 & 0.667 & 0.333 & 1 \\
\hline
\end{tabular}


Table 14. Mixer Truck Status Change Data

\begin{tabular}{|c|c|c|c|c|c|c|c|c|c|c|}
\hline \multirow{2}{*}{ Month } & \multicolumn{10}{|c|}{ Condition (Status) } \\
\hline & $\mathbf{B} / \mathbf{B}$ & $\mathbf{B} / \mathbf{r}$ & $\mathbf{B} / \mathbf{s}$ & $\mathbf{B} / \mathbf{b}$ & $\mathbf{r} / \mathbf{r}$ & $\mathbf{r} / \mathbf{s}$ & $\mathbf{r} / \mathbf{b}$ & $\mathbf{s} / \mathbf{s}$ & $\mathbf{s} / \mathbf{b}$ & $\mathbf{b} / \mathbf{B}$ \\
\hline December & 0 & 0 & 0 & 0 & 0 & 0 & 0 & $9 / 45$ & $6 / 45$ & 0 \\
\hline January & 0 & 0 & 0 & 0 & $7 / 60$ & $4 / 60$ & $4 / 60$ & 0 & 0 & 0 \\
\hline February & 0 & 0 & 0 & 0 & $3 / 60$ & $8 / 60$ & $4 / 60$ & 0 & 0 & 0 \\
\hline March & 0 & 0 & 0 & 0 & 0 & 0 & 0 & $11 / 45$ & $4 / 45$ & 0 \\
\hline April & $8 / 46$ & 0 & 0 & 0 & 0 & 0 & 0 & 0 & 0 & $7 / 29$ \\
\hline May & 0 & 0 & 0 & 0 & $3 / 60$ & $7 / 60$ & $5 / 60$ & 0 & 0 & 0 \\
\hline June & $6 / 46$ & 0 & 0 & 0 & 0 & 0 & 0 & 0 & 0 & $9 / 29$ \\
\hline July & 0 & 0 & 0 & 0 & $8 / 60$ & $2 / 60$ & $5 / 60$ & 0 & 0 & 0 \\
\hline August & 0 & $7 / 46$ & $5 / 46$ & $3 / 46$ & 0 & 0 & 0 & 0 & 0 & 0 \\
\hline September & 0 & 0 & 0 & 0 & 0 & 0 & 0 & $10 / 45$ & $5 / 45$ & 0 \\
\hline October & $10 / 46$ & 0 & 0 & 0 & 0 & 0 & 0 & 0 & 0 & $5 / 29$ \\
\hline November & $7 / 46$ & 0 & 0 & 0 & 0 & 0 & 0 & 0 & 0 & $8 / 29$ \\
\hline Sum & 0.674 & 0.152 & 0.109 & 0.065 & 0.350 & 0.350 & 0.300 & 0.667 & 0.333 & 1 \\
\hline
\end{tabular}

4.3. Calculation of Proposed Transition Probability Matrix for Production Machines

Proposed transition probability matrix I (P0) Corrective maintenance at condition 4 to determine the long-run steady state probability for each machine.

\section{a. Wheel Loader Machine}

Table 15. Transition Probability Matrix (P0) Wheel Loader Machine

\begin{tabular}{|c|c|c|c|c|}
\hline $\begin{array}{ll}\mathrm{i} & \mathrm{j} \\
\end{array}$ & 1 & 2 & 3 & 4 \\
\hline 1 & 0.250 & 0.250 & 0.250 & 0.250 \\
\hline 2 & 0 & 0.500 & 0.250 & 0.250 \\
\hline 3 & 0 & 0 & 0.500 & 0.500 \\
\hline 4 & 1 & 0 & 0 & 0 \\
\hline
\end{tabular}

\begin{tabular}{|c|c|c|c|c|c|c|c|}
\hline$\pi 1$ & & 0.250 & & 0.250 & 0.250 & 0.250 & \\
\hline$\pi 2$ & & 0 & & & 0.500 & 0.250 & 0.250 \\
\hline$\pi 3$ & $=\left[\begin{array}{llll}\pi 1 & \pi 2 & \pi 3 & \pi 4\end{array}\right]$ & 0 & & & & 0 & 0.500 \\
\hline$\pi 4$ & & 1 & 0,500 & & & 0 & \\
\hline
\end{tabular}

Note $: \pi 1+\pi 2+\pi 3+\pi 4=1$

Then the following equation will be obtained:

$$
\begin{array}{llll}
\pi 1+\quad \pi 2+\quad \pi 3+ & \pi 4 & =1 \\
0.250 \pi 1+ & & \pi 4 & =\pi 1 \\
0.250 \pi 1+0.500 & \pi 2+ & & =\pi 2 \\
0.250 \pi 1+0.250 & \pi 2+0.500 \pi 3 & & =\pi 3 \\
0.250 \pi 1+0.250 & \pi 2+0.500 \pi 3 & & =\pi 4
\end{array}
$$


The solution to the above equation is:

\begin{tabular}{|c|c|c|c|}
\hline \multirow{2}{*}{$\begin{array}{l}0.250 \pi 1+\pi 4 \\
\pi 4 \\
\pi 4 \\
\\
\\
0.250 \pi 1+0.500 \\
0.250 \pi 1 \\
0.250 \pi 1\end{array}$} & $\begin{array}{l}= \\
= \\
=\end{array}$ & \multicolumn{2}{|c|}{$\begin{array}{l}\pi 1 \\
\pi 1-250 \pi 1 \\
0.750 \pi 1\end{array}$} \\
\hline & $\begin{array}{l}\pi 2 \\
\pi 2 \\
\pi 2\end{array}$ & $\begin{array}{l}\pi 2 \\
1- \\
0.5 \\
0.2 \\
0.5\end{array}$ & $\begin{array}{ll}00 & \pi 2 \\
\pi 2 & \\
\pi 1 / 0.500\end{array}$ \\
\hline $\begin{array}{l}0.250 \pi 1+0.250 \\
0.250 \pi 1+0.250 \\
0.250 \pi 1+0.125 \\
0.375 \pi 1\end{array}$ & $\begin{array}{l}\pi 2+0.500 \pi \\
(0.5 \pi 1) \\
\pi 1\end{array}$ & $\begin{array}{l}= \\
= \\
= \\
= \\
= \\
=\end{array}$ & $\begin{array}{l}\pi 3 \\
1-0.500 \pi 3 \\
0.5 \pi 3 \\
0.5 \pi 3 \\
0.375 \pi 1 / 0.5 \\
0.75 \pi 1\end{array}$ \\
\hline $\begin{array}{l}\pi 1+\pi 2+\pi 3+\pi 4 \\
\pi 1+0.5 \pi 1+0.75\end{array}$ & $\begin{array}{r}\pi 1+0.75 \pi \\
3 \pi \\
\pi 1\end{array}$ & $\begin{array}{l}= \\
= \\
= \\
=\end{array}$ & $\begin{array}{l}1 \\
1 \\
1 \\
1 / 3=0.333\end{array}$ \\
\hline
\end{tabular}

then,

$\pi 1=0.333$

$\pi 2=0.167$

$\pi 3=0.25$

$\pi 4=0.25$

\section{b. Batching Plant Machine}

Table 16. Transition Probability Matrix (P0) Batching Plant Machine

\begin{tabular}{cccccc}
\hline $\mathrm{i}$ & $\mathrm{j}$ & 1 & 2 & 3 & 4 \\
\hline 1 & & 0.2 & 0.4 & 0.2 & 0.2 \\
\hline 2 & 0 & 0 & 0.333 & 0.667 \\
\hline 3 & 0 & 0 & 0.667 & 0.333 \\
\hline 4 & 1 & 0 & 0 & 0 \\
\hline
\end{tabular}

Obtained from the same calculation as the previous machine it is obtained, then:

$$
\begin{aligned}
& \pi 1=0.313 \\
& \pi 2=0.125 \\
& \pi 3=0.313 \\
& \pi 4=0.25
\end{aligned}
$$




\section{c. Truck Mixer Machine}

Table 17. Transition Probability Matrix (P0) for Mixer Truck Machines

\begin{tabular}{cccccc}
\hline $\mathrm{i}$ & $\mathrm{j}$ & 1 & 2 & 3 & 4 \\
\hline 1 & 0.674 & 0.152 & 0.109 & 0.065 \\
\hline 2 & 0 & 0.350 & 0.350 & 0.300 \\
\hline 3 & 0 & 0 & 0.667 & 0.333 \\
\hline 4 & 1 & 0 & 0 & 0 \\
\hline
\end{tabular}

Obtained from the same calculation as the previous machine it is obtained, then:

$$
\begin{aligned}
& \pi 1=0.469 \\
& \pi 2=0.110 \\
& \pi 3=0.269 \\
& \pi 4=0.153
\end{aligned}
$$

\begin{tabular}{|c|c|c|c|c|c|c|c|c|c|}
\hline \multirow{2}{*}{$\begin{array}{c}\text { Maintenance } \\
\text { Activities }\end{array}$} & \multicolumn{4}{|c|}{ Probabiitas P0 (Manual) } & \multicolumn{4}{|c|}{ Probabiitas P0 (POM) } & \multirow[b]{2}{*}{ Note } \\
\hline & Good & Light & Moderate & Weight & Good & Light & Moderate & Weight & \\
\hline $\begin{array}{l}\text { Wheel } \\
\text { Loader }\end{array}$ & 0.333 & 0.167 & 0.25 & 0.25 & 0.3333 & 0.1676 & 0.25 & 0.25 & Valid \\
\hline $\begin{array}{c}\text { Batching } \\
\text { Plant }\end{array}$ & 0.313 & 0.125 & 0.313 & 0.25 & 0.3124 & 0.125 & 0.3126 & 0.25 & Valid \\
\hline Truck Mixer & 0.469 & 0.110 & 0.269 & 0.153 & 0.4688 & 0.1096 & 0.2687 & 0.1528 & Valid \\
\hline
\end{tabular}

Table 18. Probability of Steady State Machines

\section{d. Calculation of Proposed Transition Probability Matrix II Production Machines}

To get a better maintenance schedule, a Markov Chain method maintenance plan is proposed which is obtained from the machine status transition probability, which is then made a proposed transition probability matrix according to the actions taken to determine the long-term steady state probability

\begin{tabular}{|c|c|c|c|c|}
\hline $\mathrm{i} \quad \mathrm{j}$ & 1 & 2 & 3 & 4 \\
\hline 1 & 0.250 & 0.250 & 0.250 & 0.250 \\
\hline 2 & 0 & 0.500 & 0.250 & 0.250 \\
\hline 3 & 0 & 1 & 0 & 0 \\
\hline 4 & 1 & 0 & 0 & 0 \\
\hline
\end{tabular}
for each machine.

1. P1 (Corrective maintenance at status 4 and preventive maintenance at status 3)

Table 19. Proposed Probability Matrix P1 Wheel Loader Machine

Obtained from the same calculation as the previous machine it is obtained
$\pi 1=0.222$
$\pi 2=0.444$
$\pi 3=0.167$
$\pi 4=0.167$

\section{P2 (Corrective maintenance at status 3, 4 and preventive maintenance at status 2)}

Table 20. Proposed Probability Matrix P2 Wheel Loader Machine

\begin{tabular}{|c|c|c|c|c|}
\hline $\mathrm{i}$ & 1 & 2 & 3 & 4 \\
\hline 1 & 0.250 & 0.250 & 0.250 & 0.250 \\
\hline 2 & 1 & 0 & 0 & 0 \\
\hline 3 & 1 & 0 & 0 & 0 \\
\hline 4 & 1 & 0 & 0 & 0 \\
\hline
\end{tabular}


Obtained from the same calculation as the previous machine it is obtained

$$
\pi 1=0.571 \quad \pi 2=0.143 \quad \pi 3=0.143 \quad \pi 4=0.143
$$

3. $P 3$ (Corrective maintenance at status 4 and preventive maintenance at status 2,3 )

Table 21. Proposed Probability Matrix of Wheel Loader Machine P3

\begin{tabular}{|c|c|c|c|c|}
\hline i $j$ & 1 & 2 & 3 & 4 \\
\hline 1 & 0.250 & 0.250 & 0.250 & 0.250 \\
\hline 2 & 1 & 0 & 0 & 0 \\
\hline 3 & 0 & 1 & 0 & 0 \\
\hline 4 & 1 & 0 & 0 & 0 \\
\hline
\end{tabular}

Obtained from the same calculation as the previous machine it is obtained $\pi 1=0.5 \quad \pi 2=0.25 \quad \pi 3=0.125 \quad \pi 4=0.125$

\section{P4 (Corrective maintenance at status 3, 4)}

Table 22. Proposed Probability Matrix of P4 Wheel Loader Machine

\begin{tabular}{|c|c|c|c|c|}
\hline $\begin{array}{ll}\mathrm{i} & \mathrm{j} \\
\end{array}$ & 1 & 2 & 3 & 4 \\
\hline 1 & 0.250 & 0.250 & 0.250 & 0.250 \\
\hline 2 & 0 & 0.500 & 0.250 & 0.250 \\
\hline 3 & 1 & 0 & 0 & 0 \\
\hline 4 & 1 & 0 & 0 & 0 \\
\hline
\end{tabular}

Obtained from the same calculation as the previous machine it is obtained

$$
\pi 1=0.444 \quad \pi 2=0.222 \quad \pi 3=0.167 \quad \pi 4=0.167
$$

For the calculation of the batching plant and truck mixer machines are the same as the calculation process for the wheel loader machine and the only difference is the probability matrix so that the following results are obtained.

\begin{tabular}{|c|c|c|c|c|c|c|c|c|c|}
\hline \multirow{2}{*}{$\begin{array}{c}\text { Maintenance } \\
\text { Activities }\end{array}$} & \multicolumn{4}{|c|}{ Probabiitas (Manual) } & \multicolumn{4}{|c|}{ Probabiitas ( POM) } & \multirow[b]{2}{*}{ Ket } \\
\hline & Good & Light & Moderate & Weight & Good & Light & Moderate & Weight & \\
\hline P1 & 0.222 & 0.444 & 0.167 & 0.167 & 0.2222 & 0.4444 & 0.1667 & 0.1667 & Valid \\
\hline $\mathrm{P} 2$ & 0.571 & 0.143 & 0.143 & 0.143 & 0.5714 & 0.1429 & 0.1429 & 0.1429 & Valid \\
\hline P3 & 0.5 & 0.25 & 0.125 & 0.125 & 0.5 & 0.25 & 0.125 & 0.125 & Valid \\
\hline $\mathrm{P} 4$ & 0.444 & 0.222 & 0.167 & 0.167 & 0.4444 & 0.2222 & 0.1667 & 0.1667 & Valid \\
\hline
\end{tabular}

Table 23. Probability of Wheel Loader Engine Steady State

\begin{tabular}{|c|c|c|c|c|c|c|c|c|c|}
\hline \multirow{2}{*}{$\begin{array}{c}\text { Maintenance } \\
\text { Activities }\end{array}$} & \multicolumn{4}{|c|}{ Probabiitas (Manual) } & \multicolumn{4}{|c|}{ Probabiitas (POM ) } & \multirow[b]{2}{*}{ Ket } \\
\hline & Good & Light & Moderate & Weight & Good & Light & Moderate & Weight & \\
\hline P1 & 0.313 & 0.282 & 0.157 & 0.25 & 0.3126 & 0.2812 & 0.1562 & 0.2501 & Valid \\
\hline $\mathrm{P} 2$ & 0.556 & 0.222 & 0.111 & 0.111 & 0.5556 & 0.2222 & 0.1111 & 0.1111 & Valid \\
\hline $\mathrm{P} 3$ & 0.5 & 0.3 & 0.1 & 0.1 & 0.5 & 0.3 & 0.1 & 0.1 & Valid \\
\hline $\mathrm{P} 4$ & 0.455 & 0.182 & 0.152 & 0.213 & 0.4546 & 0.1818 & 0.1515 & 0.2122 & Valid \\
\hline
\end{tabular}

Table 24. Probability of Steady State Machine Batching Plant 
Table 25. Probability of Steady State Mixer Truck Machine

\begin{tabular}{|c|c|c|c|c|c|c|c|c|c|}
\hline \multirow{2}{*}{$\begin{array}{c}\text { Maintenance } \\
\text { Activities }\end{array}$} & \multicolumn{4}{|c|}{ Probabiitas (Manual) } & \multicolumn{4}{|c|}{ Probabiitas (POM ) } & \multirow[b]{2}{*}{ Ket } \\
\hline & Good & Light & Moderate & Weight & Good & Light & Moderate & Weight & \\
\hline P1 & 0.383 & 0.333 & 0.159 & 0.125 & 0.3832 & 0.3334 & 0.1585 & 0.1249 & Valid \\
\hline $\mathrm{P} 2$ & 0.754 & 0.115 & 0.082 & 0.049 & 0.7542 & 0.1146 & 0.0822 & 0.049 & Valid \\
\hline P3 & 0.697 & 0.182 & 0.076 & 0.045 & 0.6969 & 0.1819 & 0.076 & 0.0453 & Valid \\
\hline $\mathrm{P} 4$ & 0.641 & 0.15 & 0.122 & 0.087 & 0.6411 & 0.1499 & 0.1224 & 0.0866 & Valid \\
\hline
\end{tabular}

After getting the steady state probability, the next step will be compared with the calculation results using the POM application. If the results are valid, then it will continue with the next step, namely the calculation of costs and calculations for scheduling machine maintenance.

\subsection{Maintenance Cost Calculation}

In calculating machine maintenance costs, corrective and preventive maintenance time data are needed as well as corrective and preventive maintenance cost data.

a. Wheel Loader Machine

$=$ Corrective maintenance costs $=$ corrective maintenance time $\times$ corrective downtime cost

$=74.67$ hour $/$ year X Rp. $295,500.00=$ Rp. $22,064,000$

$=$ Preventive maintenance costs $=$ preventive maintenance time $\times$ preventive downtime costs

$=10.25$ hour $/$ year X Rp. $294,341.46=$ Rp. 3,017,000

Company maintenance costs $=$ Corrective maintenance costs + Preventive maintenance costs

$=$ Rp. $22,064,000+$ Rp. $3,017,000=$ Rp. $25,081,000$

b. Batching Plant Machine

$=$ Corrective maintenance costs $=$ corrective maintenance time $\times$ corrective downtime cost

$=88.5$ hour $/$ year X Rp. $236,316.38=$ Rp. $20,914,000$

$=$ Preventive maintenance costs $=$ preventive maintenance time $\times$ preventive downtime costs

$=11.25$ hour $/$ year X Rp. $185,955.56=$ Rp. $2,092,000$

Company maintenance costs $=$ Corrective maintenance costs + Preventive maintenance costs

$=$ Rp. $20,914,000+$ Rp. $2,092,000=$ Rp. $23,006,000$

c. Truck Mixer Machine

$=$ Corrective maintenance costs $=$ corrective maintenance time $\times$ corrective downtime cost

$=102.17$ hour $/$ year X Rp. $399,187.63=$ Rp. $40,785,000$

$=$ Preventive maintenance costs $=$ preventive maintenance time $\times$ preventive downtime costs

$=15.5$ hour $/$ year X Rp. 106,967.74=Rp. $1,658,000$

Company maintenance costs $=$ Corrective maintenance costs + Preventive maintenance costs

$=$ Rp. $40,785,000+$ Rp. $1,658,000=$ Rp. $42,443,000$

The total cost of maintaining the 3 machines incurred by the company during December 2018 November 2019 is Rp. $25,081,000+$ Rp. 23,006,000 + Rp. 42,443,000 = Rp. 90,530,000 


\subsection{Maintenance Proposed I (Tc2)}

Expected average cost of maintenance of proposal I (corrective maintenance at status 4) using the markov chain method.

Wheel Loader Machine

Expected average cost $=$ steady state probability $\times$ corrective maintenance cost

$\mathrm{Po}=0.333(0)+0.167(0)+0.25(0)+0.25($ Rp. 22.064.000)

$\mathrm{Po}=\mathrm{Rp} 5,516,000$

For batching plant machines and mixer trucks, the calculation uses the same formula as wheel loader machines so that the calculation results are as follows.

Total cost of maintenance proposal I

$=$ wheel loader machine + batching plant machine + truck mixer engine

$=\operatorname{Rp~5,516,000+Rp~5,228,500+Rp~6,240,105~}=\operatorname{Rp~16,984,605~}$

From the calculations, it can be seen that the company's total maintenance costs amounted to Rp. $16,984,605$

\subsection{Maintenance Proposed II (Tc3)}

From the four proposals, the total cost of maintenance with the markov chain method will be obtained from December 2018 to November 2019.

Wheel Loader Machine

- $P 1$ (Corrective maintenance at status 4 and preventive maintenance at status 3 )

$=0.222(0)+0.444(0)+0.167(\operatorname{Rp} 3,017,000)+0.167(\operatorname{Rp} 22,064,000)=\operatorname{Rp} 4,188,527$

- $P 2$ (Corrective maintenance at status 3, 4 and preventive maintenance at status 2)

$=0.571(0)+0.143(\operatorname{Rp~3,017,000)}+0.143(\operatorname{Rp~22,064,000)}+0,143($ Rp. 22,064,000)

$=\operatorname{Rp} 6.741 .735$

- $P 3$ (Corrective maintenance at status 4 and preventive maintenance at status 2, 3)

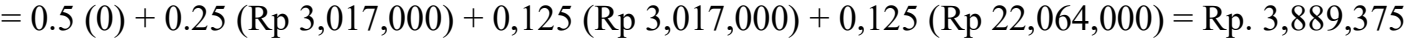

- $P 4$ (Corrective maintenance at status 3 and 4$)$

$=0.444(0)+0.222(0)+0.167(\operatorname{Rp} 22,064,000)+0.167(\operatorname{Rp} 22,064,000)=\operatorname{Rp} 7,369,376$

From the calculations, the lowest average cost of expectations lies in the proposed maintenance $P 3$ (corrective maintenance at status 4 and preventive maintenance at status 2, 3) of IDR 3,889,375

For batching plant machines and mixer trucks, the calculation uses the same formula as wheel loader machines so that the calculation results are as follows.

Total cost of maintenance proposal II

$=$ wheel loader machine + batching plant machine + truck mixer engine

$=\operatorname{Rp~3,889,375+Rp~2,928,200+Rp~2,263,089~=~Rp~9,080,664~}$

From the calculations, it can be seen that the company's total maintenance costs of Rp 9.080 .664

\subsection{Cost Savings}

After reviewing the cost of maintaining the condition of the company (Tc1) in December 2018 to November 2019 for all identified production machines, the average expected cost of maintenance of proposal I (Tc2), and the average cost of expected minimum maintenance of maintenance of proposal II (Tc3) in Steady State from December 2018 to November 2019 for all machines identified. Then the table is created as show in Table 26.

\subsection{Machine Maintenance Scheduling Planning Using the Markov Chain Method}

Maintenance of damage to wheel loaders, batching plants and mixer trucks in the company's condition in 12 months takes 265.34 hours obtained from the sum of total corrective maintenance times for wheel loader engines, batching plants and mixer trucks. 
Table 26. Maintenance Cost Savings

\begin{tabular}{|c|c|c|}
\hline & $\begin{array}{c}\text { Total maintenance costs } \\
\text { (wheel loader, batchung } \\
\text { plant, truck mixer) }\end{array}$ & Savings (from company \\
\hline $\begin{array}{c}\text { Maintenance Cost for } \\
\text { Company Condition (Tc1) }\end{array}$ & Rp. $90,530,000$ & \\
\hline $\begin{array}{l}\text { Expected maintenance costs } \\
\text { for proposal I }(T c 2)\end{array}$ & Rp. $16,984,605$ & Rp. 73,545,395 (81.24\%) \\
\hline $\begin{array}{l}\text { Expected maintenance costs } \\
\text { for proposal II }(T c 3)\end{array}$ & Rp. 9,080,664 & Rp. 81,449,336 (89.97\%) \\
\hline
\end{tabular}

Table 27. Corrective Maintenance Time Data

\begin{tabular}{clc}
\hline No & \multicolumn{1}{c}{ Machine Type } & Corrective Maintenance (hours / year) \\
\hline 1 & Wheel Loader & 74.67 \\
\hline 2 & Batching Plant & 88.5 \\
\hline 3 & Truck Mixer & 102.17 \\
\hline & Total & 265.34 \\
\hline
\end{tabular}

Maintenance of machines identified for the condition of the company and maintenance of proposal I using the markov chain method for 1 year require the following time:

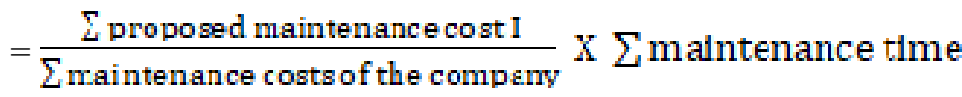

$=(\operatorname{Rp} 16,984,605) /(\operatorname{Rp} 90,530,000) \times 265.34$ hours

$=49.78$ hours $=50$ hours

So engine maintenance takes 50 hours and requires a maintenance fee of Rp. 16,984,605 with maintenance scheduling planning as follows:

Maintenance schedule planning for wheel loader engines:

$$
\begin{aligned}
& =\frac{\text { Eproposed maintenance cost I }}{\sum \text { maintenance costs of the compeny }} X \sum \text { malntenance time } \\
& =(\operatorname{Rp~} 16,984,605) /(\operatorname{Rp} 90,530,000) X 74.67 \text { hours } \\
& =14.00843742 \text { hours }=14.009 \text { hours }
\end{aligned}
$$

So maintenance is carried out every 14.009 hours

The maintenance schedule planning for the batching plant and truck mixer machines is obtained using the same calculation steps so that the following results are obtained. For batching plant machines, maintenance is carried out every 16.604 hours, for mixer truck machines, maintenance is carried out every 19.168 hours. And the second proposal maintenance scheduling uses the same formula. The results are that for wheel loader machines, maintenance is carried out every 7,490 hours, for batching plant machines maintenance is carried out every 8,877 hours, for mixer truck machines maintenance is carried out every 10,248 hours

\section{Conclusion}

The conclusions obtained based on the research are as follows:

For proposal I, it will take 49.78 hours $=50$ hours and the budget spent is Rp. 16,984,605. Each maintenance process such as a wheel loader every 14.00843742 hours $=14.009$ hours, a batching plant 
machine every 16.60375061 hours $=16.604$ hours, a truck mixer engine every 19.16842033 hours $=$ 19.168 hours. For the second proposal scheduling takes 26.62 hours $=27$ hours and the budget spent is Rp. 9,080,664. Maintenance process for each machine such as wheel loader every 7,489483177 hours $=7,490$ hours, batching plant machines every 8,877043676 hours $=8,877$ hours, truck mixer machines every 10.24822093 hours $=10.248$ hours.

The policy implemented in proposal I is corrective maintenance at status 4 (major damage). For each machine, such as a wheel loader, maintenance costs are IDR 5,516,000, a batching plant machine costs IDR 5,228,500 and a truck mixer engine maintenance costs IDR 6,240,105.

There are 4 policies implemented in proposal II, namely:

- $\quad P 1$ (Corrective maintenance at status 4 and preventive maintenance at status 3 )

- $\quad P 2$ (Corrective maintenance at status 3, 4 and preventive maintenance at status 2)

- $\quad P 3$ (Corrective maintenance at status 4 and preventive maintenance at status 2,3 )

- $\quad P 4$ (Corrective maintenance at status 3 and 4 )

Of the 4 policies, the expected maintenance costs for each machine will be generated. Wheel loader engine maintenance expectations for P1 = IDR 4,188,527, P2 = IDR 6,741,735, P3 = IDR 3,889,375, $\mathrm{P} 4=$ IDR 7,369,376. Batching plant machine maintenance expectations for P1 = Rp. 5,556,944, P2 = Rp. 5,107,332, P3 = Rp. 2,928,200, P4 = Rp. 7,633,610. Truck mixer engine maintenance expectations for P1 = IDR 5,361,747, P2 = IDR 5,533,505, P3 = IDR 2,263,089, P4 = IDR 8,483,280. Of the 4 policies chosen are those that have the lowest expected cost of maintenance to be proposed in the company, namely P3.

The resulting savings after calculating expected maintenance costs when compared with company costs are as follows:

- The expected maintenance cost for proposal I is Rp. 16,984,605 when compared to the costs incurred by the company of Rp. 90,530,000 then the cost savings are Rp. 73,545,395 (81.24\%)

- Expected maintenance cost for proposal II of Rp. 9,080,664 when compared to the costs incurred by the company of Rp. 90,530,000 then the cost savings are Rp. 81,449,336 (89.97\%).

After conducting the research, below are suggestions that will be submitted by researchers, including:

1. After performing various data processing calculations the proposal to be given to the company is proposal II because it has the smallest expected maintenance cost (Rp. 9,080,664) and has cost savings when compared to the company (Rp. 90,530,000), proposal II has The biggest savings were (Rp. 81,449,336 (89.97\%)) with the machine maintenance policy, namely P3 (corrective maintenance at status 4 and preventive maintenance at status 2,3). By scheduling each machine such as wheel loader every 7,489483177 hours $=7,490$ hours, batching plant machines every 8,877043676 hours $=8,877$ hours, truck mixer machines every 10.24822093 hours $=10,248$ hours.

2. Based on the results of policy research that must be carried out in order to meet the requirements for cost estimation, the company must carry out corrective maintenance at status 4 and preventive maintenance at status 2,3 , where the company must immediately replace components or spare parts if the production machine is severely damaged in addition to Therefore, the company must take preventive or preventive maintenance measures if the machine experiences minor and moderate damage so that the machine does not change its damaged status to become a serious one.

3. Proposals submitted to PT. DEWI RATIH PUTERA can be used as a reference in designing engine maintenance scheduling in order to minimize maintenance costs.

4. Machine maintenance proposals that the company must do are divided into:

- Corrective maintenance where the company must replace spare parts or engine components that have been badly damaged and cannot be used anymore

- Preventive maintenance where the company must take precautions so that the machine does not suffer more serious damage. The actions taken are adjusted to the type of machine to be treated, for example:

1. Wheel loader

a. Hydraulic ass seal cleaning

b. Air filter cleaning, fuel filter

c. Addition of coolant, hydraulic oil, battery water

2. Batching plant 
a. Cleaning of residual cement on hydraulic doors

b. Water cleaning in the compressor tube

c. Hydraulic as seal cleaning

d. Addition of Gearbox oil, hydraulic oil

3. Truck mixer

a. Air filter cleaning, fuel filter

b. Tire rotation

c. The addition of brake oil, coolant, battery water

\section{References}

[1] R. T. Hartanto, "Perencanaan Pemeliharaan Mesin Pompa Gilingan Saus Dengan Metode Markov Chain Untuk Minimasi Biaya Pemeliharaan (Studi Kasus : PT. Lombok Gandaria, Unit Maintenance)," Unpublished thesis, 2014.

[2] R. R. Sinabariba, "Penerapan Metode Markov Chain dalam Perencanaan Perawatan Mesin Untuk Meminimumkan Biaya Perawatan (Studi kasus: PTPN IV Unit Usaha Aldolina)" Unpublished thesis, 2017.

[3] I. Irdianto, "Penggunaan Metode Markov Chain Dalam Penjadwalan Perawatanmesin Untuk Meminimalkan Biaya Kerusakan Mesin dan Perawatan Mesin Mill 303," Jurnal Teknik, vol. 2, pp. 11-17, September 2019.

[4] D. S. Maulana, F. Handoko, and E. Adriantantri, "Perencanaan Perawatan Mesin Dengan Menggunakan Metode Markov Chain Di PT. Karyamitra Budisentosa," Unpublished thesis, 2018 .

[5] D. I. Permatasari, S. S. Dahda, and M. Z. Fathoni, "Perencanaan Perawatan Mesin Filling dengan Metode Markov Chain Untuk Meminimumkan Biaya Perawatan di PT. Swabina Gatra," Matrik", vol. 678, pp. 1-11, 2018.

[6] E. Pudji and I. Fahma, "Perencanaan Pemeliharaan Mesin dengan Menggunakan Metode Markov Chain Untuk Mengurangi Biaya Pemeliharan di PT. Philips Indonesia.” Thesis, 2012.

[7] M. Hartono and I. Mas'udin, "Perencanaan Perawatan Mesin Dengan Metode Markov Chain Guna Menurunkan Biaya Perawatan", 2012.

[7] D. G. Allo, D. Hatidja dan M. Paendong. "Analisis Rantai Markov Untuk Mengetahui Peluang Perpindahan Merek Kartu Seluler Pra Bayar GSM", Jurnal MIPA UNSRAT ONLINE, vol. 2, no. 1, pp. 17-22, 2012.

[8] H. Anton, and C. Rorres, Aljabar Linear Elementer versi Aplikasi. Edisi ke-8, jilid 2. Terjemahan Izham Harmein dan Julian Gresdando. Jakarta: Erlangga, 2004.

[9] W. K. Ching and K. Ng. Michael, "Markov Chain Models, Algorithm and Aplication." New York: Springer, 2006.

[10] I. Djan, dan R. Ruvendi, "Prediksi perpindahan penggunaan merek handphone di kalangan mahasiswa (studi kasus pada mahasiswa STIE Binaniaga)", Jurnal Ilmiah Binaniaga, vol. 2, no. 1,2006

[11] F. S. Hiller, and G. J. Lieberman. "Introduction to Operation Research Eight Edition Jilid 2", Jogjakarta: Penerbit Andi, 2008.

[12] Y. Langi, "Penentuan Klasifikasi State Pada Rantai Markov Dengan Menggunakan Nilai Eigen dari Matriks Peluang Transisi", Jurnal Ilmiah Sains, vol. 11, no. 1, pp.124-130, 2011.

[13] Mamonto, S. Y. Langi, and A. Rindengan, "Penerapan Hidden Markov Model Pada Harga Saham", Jurnal De Cartesian, vol. 5, no. 1, pp. 35-41, 2016.

[14] S. Mulyono, "Riset Operasi. Fakultas Ekonomi Universitas Indonesia", Jakarta, 2007.

[15] Nurjana, S. M. Paendong, and Y. Langi, "Penerapan Rantai Markov Dalam Pemilihan Minat Masuk Siswa SMA Ke Universitas Di Indonesia”, Jurnal De Cartesian, vol. 5, no. 1, pp. 50-56, 2016.

[16] Siswanto, Operations Research. Jakarta: Erlangga, 2007

[17] Tumilaar, K. Y. Langi, and A. Rindengan. "Hidden Markov Model," Jurnal De Cartesian, vol. 4, no. 1, pp. 86-94, 2015. 